\title{
DEVELOPING NMR PROBES OF THE MICROSCOPIC WATER DISTRIBUTION IN FOOD
}

\author{
Brian Hills, Aude Gravelle, Kevin Wright, Niusa Marigheto and David Hibberd \\ Institute of Food Research, Norwich Research Park, Colney, Norwich NR47UA, \\ Email: brian.hills@bbsrc.ac.uk
}

\begin{abstract}
We briefly review the current status of multidimensional NMR relaxation and diffusion studies of the microscopic water distribution in complex multiphase, multicomponent food systems, including a detailed discussion of micro-phase separated mixed biopolymer systems and water compartmentation in cellular plant tissue. We propose a number of novel pulse sequences for the ultrafast acquisition of the multidimensional relaxation spectra.
\end{abstract}

\section{Introduction}

Research on structure-function relations in food naturally focuses on the biopolymers comprising the food matrix and their interactions with sugars, lipids, preservatives and flavours. Yet water also has an essential role in almost every aspect of food science, including the processing response of raw materials, food stability, its organoleptic properties as well as its microbial safety. It is therefore surprising that, despite thousands of research publications, our fundamental understanding of the role of water in food remains largely empirical. Part of the problem lies in the extreme complexity of most foods, which, being micro-heterogeneous, multi-component and multi-phase systems makes it hard to predict how water partitions between the various components and microphases. There are even greater problems predicting water transport during complex processing operations like extrusion cooking, baking, drying, rehydration and freezing. Nor do the difficulties occur only during processing. Operations such as baking and air-drying invariably create nonequilibrium distributions of water that slowly readjust during storage. This slow water redistribution is often associated with undesired textural changes, such as staling, and reduced food shelf life. Of course, similar problems occur during the storage of non-processed foods like fruit and vegetables. Here it is the (sub-)cellular redistribution of water between membrane-bound compartments that affects quality though altered turgor pressure, accelerated spoilage reactions and tissue breakdown.

Water transport on the macroscopic distance scale during processes such as drying can be measured non-invasively with Magnetic Resonance Imaging $^{1}$ or, destructively, by mechanical slicing and weighing. The experimental macroscopic water concentration profiles can usually be modelled with conventional mass-transfer formalisms based on diffusion theory, two-film theory and penetration theory described in standard chemical engineering texts ${ }^{2,3}$. Invariably, these macroscopic mass-transfer formalisms introduce empirical transport coefficients such as effective water diffusion coefficients and interfacial mass transfer coefficients that have a complicated, and usually poorly characterised, functional dependence on the timeand space-dependent local water content, temperature, pressure and composition. While it is possible, in simple systems, to measure this functional dependence the real scientific challenge lies in understanding how these macroscopic water transport 
coefficients are determined by the changing matrix microstructure, the changing microscopic water distribution and, ultimately, by water-biopolymer-solute interactions at the molecular level. This is an increasingly urgent task because biopolymer structure and microstructure can now be engineered, or, at least modified, by an impressive array of genetic, enzymatic, chemical and mechanical techniques, but if we do not understand how the modifications alter the microscopic and molecular water distribution then we have no way of predicting how the modified biopolymer or matrix microstructure will affect functional behaviour such as texture and shelf-life.

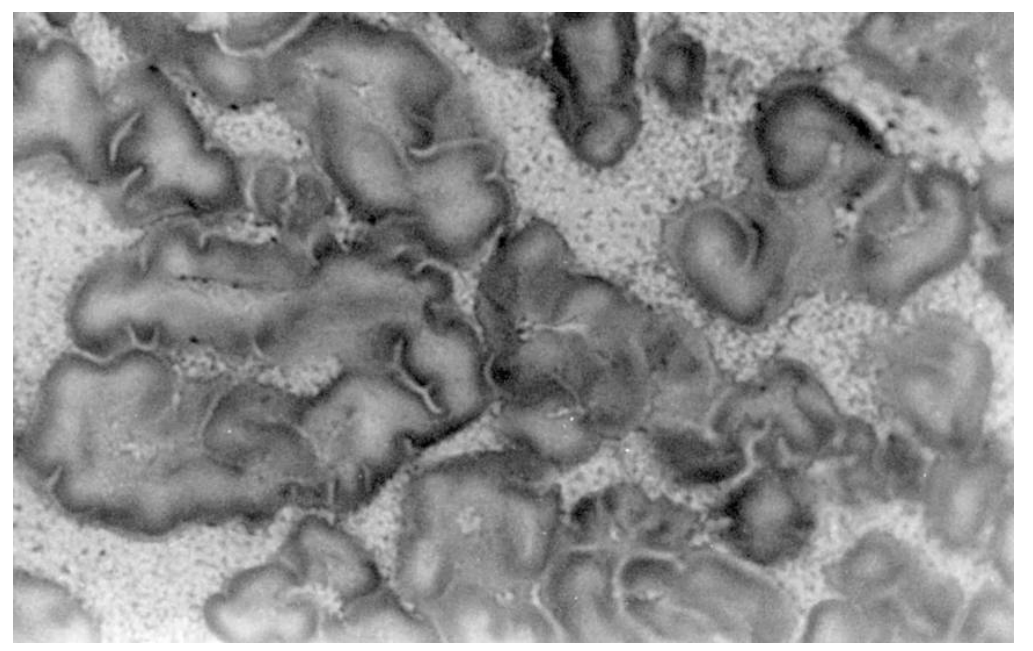

Figure 1. Partially heat-gelatinised pea starch granules embedded in an egg albumin protein matrix. Courtesy of Dr Mary Parker.

Many of the factors affecting water distribution on the microscopic distance scale are illustrated by the thermal processing of a model system comprising pea starch granules embedded in an egg albumin matrix. This system has at least two microphases and three biopolymer components (amylose, amylopectin and albumin) so it is representative of many microheterogeneous foods. The two microphases are clearly seen in the optical micrograph in figure 1 which shows the system after it has been heated and allowed to cool. Although the micrographs do not show the water distribution we expect that as the temperature is first raised the native starch granules swell and draw water out of the albumin phase until the starch gelatinisation temperature of about $60^{\circ} \mathrm{C}$ is reached. Thereafter, we expect the release of amylose from the granules to carry water back into the albumin phase where there is microscopic phase separation between the amylose and albumin mixed polymer phases. If the temperature is further raised the proteins in the egg albumin phase begin to denature and cross-link and bind water. There is therefore a backwards and forwards movement of water between microphases on the microscopic distance scale. The amount of water migration determines the gelatinisation temperature and the rate of phase separation, so it is a prime factor in controlling the processing response in this system. Until the factors controlling this redistribution are understood we cannot predict the effects of modifying the structure of the starch granules or the effect of altering the composition of the albumin phase. Moisture migration between components and microphases during storage also affects shelf life by altering rates of starch retrogradation and phase separation and therefore, ultimately, texture and microbial stability. 
In principle the water distribution can be calculated from knowledge of the sorption isotherms of each microphase because, at thermodynamic equilibrium, the water chemical potential is everywhere equal so that the water activity is also everywhere equal. However, in practice, this is not always so straightforward because, as the starch-albumin system illustrates, the composition of each microphase can also change with temperature, so a plethora of sorption isotherms would be needed. Moreover it is not necessarily safe to assume thermodynamic equilibrium at all stages because such factors as polymer entanglement, surface barriers to moisture migration and glassy state formation may kinetically arrest the system. Ultimately, therefore, it is necessary to measure the changing water distribution but finding suitable experimental tools for monitoring water (re-)distribution between different phases and components on the microscopic distance scale continues to be a challenge and this will be the focus of the remaining sections.

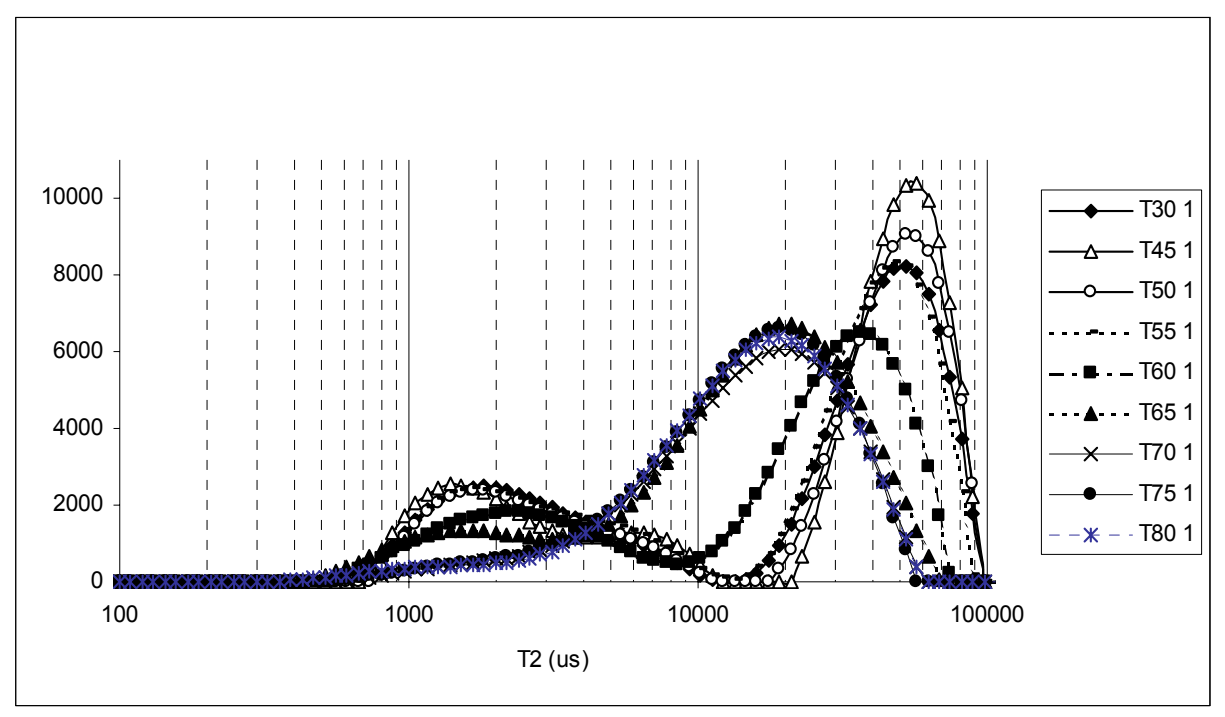

Figure 2. The relaxation spectrum of a 2-phase system comprising potato starch granules embedded in a BSA protein matrix and heated to the indicated temperatures.

\section{Experimental probes of the microscopic water distribution}

While microscopic techniques such as optical and electron microscopy and AFM, give detailed information about the microstructure of the food matrix, as exemplified by figure 1, they fail to show how water is distributed between the microphases. NMR is undoubtedly the most promising experimental tool for doing this, but even the NMR methods are problematic and require further development. In some simple cases the one-dimensional distribution of water proton transverse relaxation times, the so-called "relaxation spectrum" obtained by constrained inverse Laplace transformation of the CPMG echo decay envelope ${ }^{1}$ gives useful information about microscopic water distribution. Figure 2 shows the relaxation spectrum for a suspension of potato starch granules in a $30 \%$ BSA solution as a function of thermal processing temperature. In this experiment, which was designed to mimic the system in figure 1, the starch suspension was heated to the indicated temperature for 20 minutes, then cooled to $23^{\circ} \mathrm{C}$, where it was measured with the CPMG pulse sequence with a 90-180 pulse spacing of $240 \mu$ s. The resulting decay curve was analysed as a continuous distribution of exponentials using the standard Resonance Instruments 
WINDXP software. Each peak in figure 2 corresponds to water in a different microphase, though it is not always straightforward to assign them to specific structures. The peak at ca $50 \mathrm{~ms}$ in the unheated sample is believed to correspond to water in the BSA solution outside the granules and the broad peaks between 1-10ms to water inside the starch granules ${ }^{4,5}$. Heating the sample at or above the gelatinisation temperature of ca. 60 degrees causes the release of amylose and water from the granule into the surrounding BSA phase and this can be quantified as an increase in the area of the peaks above ca $6 \mathrm{~ms}$.

Difficulties in peak assignment and peak overlap are two obvious shortcomings of this approach, as is the unknown extent to which water diffusion between different microphases averages the signal. The later can be minimised by working at temperatures just above freezing, and peak separation can be altered to some extent by modifying the pulse spacing and by varying the extent of diffusive attenuation by imposition of constant field gradients during the CPMG acquisition. Alternatively, deuterium relaxometry in a $\mathrm{D}_{2} \mathrm{O}$ exchanged system could give insight by removing the signal from non-exchanging biopolymer protons.

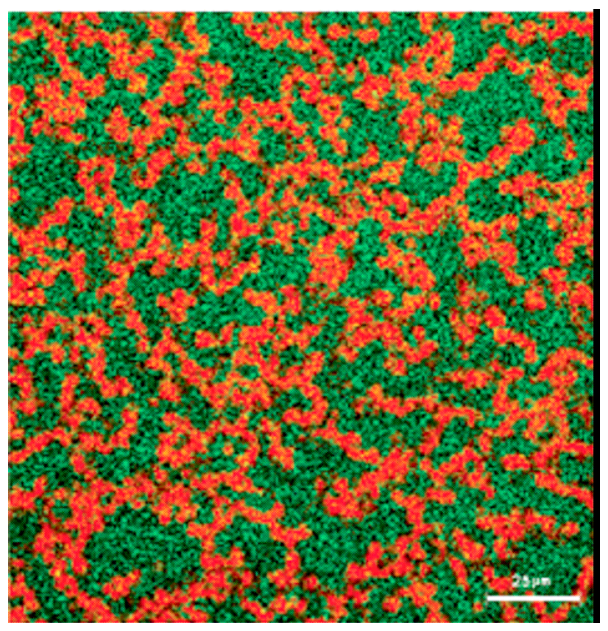

Figure 3. An optical micrograph of a phase separated mixture of $2 \% B S A$ and $0.43 \%$ pectin that has been heated at $80^{\circ} \mathrm{Cfor} 30$ minutes. Taken from reference 6.

Phase-separating biopolymer mixtures illustrate another related problem common to complex food systems, namely, that it is extremely difficult to determine not just the water content, but also the biopolymer composition of each microphase. Figure 3 taken from reference 6 shows a phase-separated BSA-pectin mixture with BSA-rich and pectin-rich microphases having spatial correlation lengths of the order of 20 microns. While the optical micrographs beautifully illustrate the microphase structure, they fail to give information on the biopolymer composition of the two microphases or on their water content. To try to tackle these questions we extended the 1-dimensional CPMG method illustrated in figure 2 to 2-dimensions using $T_{1}-T_{2}$ correlation spectroscopy ${ }^{7}$. Even with spatial correlation lengths of the order of 20 microns it was unclear at the outset whether 2-dimensional NMR methods would give separate water peaks for the two microphases. This is because fast diffusion of water between microphases on the NMR measurement timescale of a few milliseconds averages the signal so that the relaxation time peaks merge together. In a time, $t$, water molecules in pure water diffuse a distance $(6 \mathrm{Dt})^{1 / 2}$ which is about $10 \mu \mathrm{m}$ in ten milliseconds, which is a typical NMR measurement timescale. This 
means that, unless there a barrier to water diffusion, the NMR relaxation method has a spatial resolution limit of about 10 microns. We are therefore in a "borderline" situation where partial diffusive averaging of the water peaks in the phase separated system of figure 3 is to be expected.

The system was first calibrated by acquiring the $\mathrm{T}_{1}-\mathrm{T}_{2}$ spectrum (figure $4 \mathrm{a}$ ) of a sample where a $1 \%$ pectin solution was layered on top of an equal volume of an $8 \%$ BSA solution that had been previously gelled by heating to $80^{\circ} \mathrm{C}$ for 30 minutes.
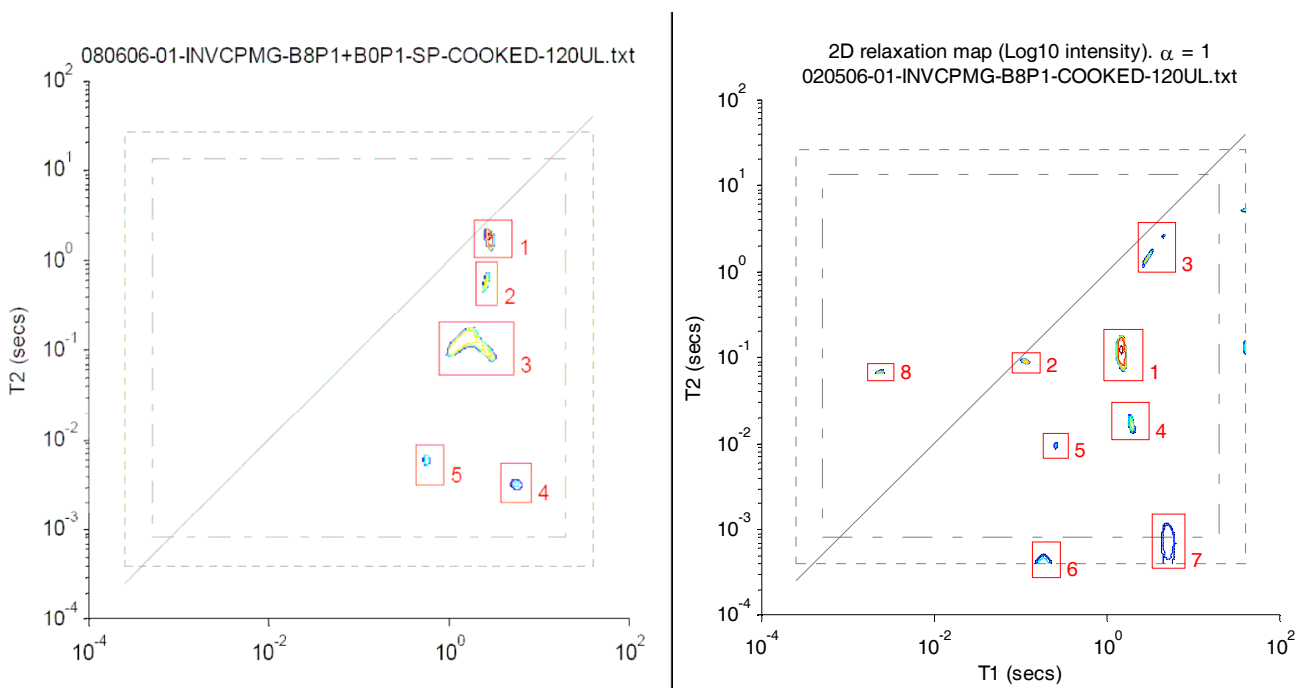

Figure 4. $T_{1}-T_{2}$ spectra of Left) 2 layers comprising $8 \% B S A-1 \%$ pectin (bottom layer) and $1 \%$ pectin (top layer) Right) microphase separated mixture of $8 \% B S A$ and $1 \%$ Pectin. Both samples heated to $80^{\circ} \mathrm{C}$ for 30 minutes.

Although there are many unassigned peaks arising from $\mathrm{CH}$ proton pools in the 2 biopolymers the dominant peaks arise from water in the pectin phase (peak 1, Left spectrum 43.6\%) and in the BSA gel (peak 3, Left spectrum 45.6\%). Having characterised the separate water peaks of the macroscopically separate phases we proceeded to see whether separate peaks could also be resolved in the micro-phase separated mixed biopolymer system. Figure $4 \mathrm{~b}$ shows the $\mathrm{T}_{1}-\mathrm{T}_{2}$ spectrum of a mixed system obtained by heating an $8 \%$ BSA and $1 \%$ pectin mixture. Peaks 1 and 3 can still be resolved confirming the existence of distinct microphases in the mixed system with relative intensities (peak 1 pectin-rich 1.8\%; peak 3 BSA-rich 93.2\%). However, as anticipated, the relaxation times of the BSA-rich peak are the same as those of a pure $8 \%$ BSA gel, so there must be considerable diffusion of water between the microphases and the indicated percentages cannot reflect the true micro-phase composition. It would therefore appear that the separation of the water-dominated peaks in the $T_{1}-T_{2}$ spectrum will only succeed in quantitatively characterising the microphases if the domain size is sufficiently large to prevent fast water diffusion between the phases, which, in water-rich systems probably means domain sizes of the order of 100 microns or more. In principle, this limitation could be removed if the biopolymer-specific peaks in the $T_{1}-T_{2}$ spectrum showed shifts related to microphase composition. However, perusal of figures 4 shows that most of the biopolymer peaks, which presumably arise from non-exchanging $\mathrm{CH}$ protons, are of very low intensity compared to the dominant water peak which suggests that some form of watersuppression is required before this idea can be tested. 

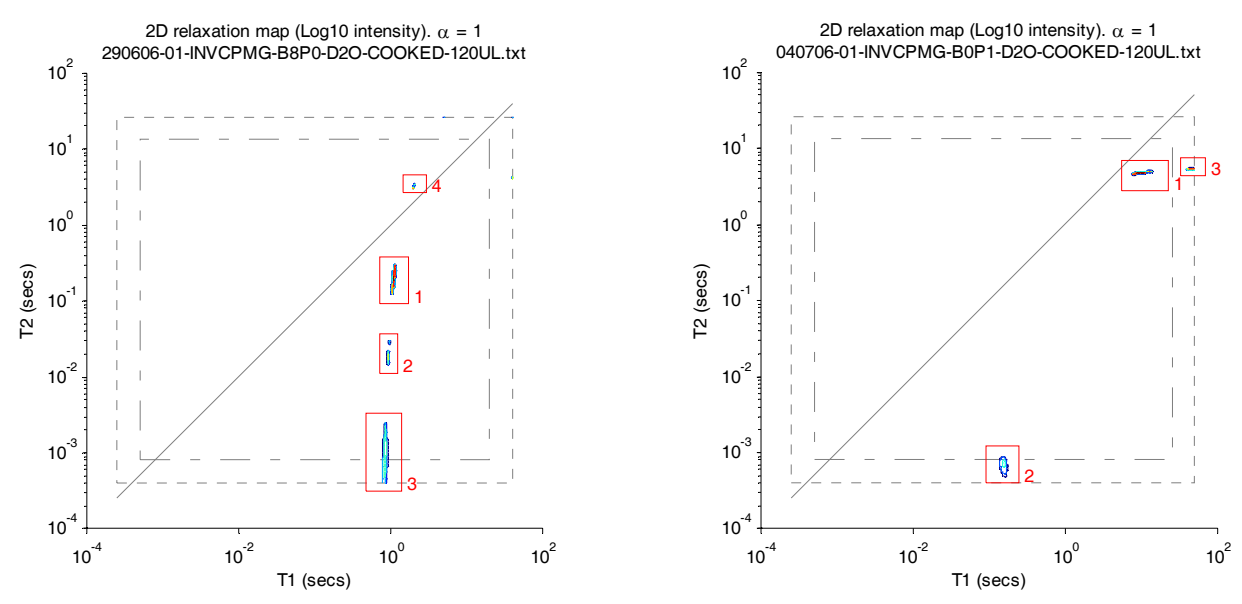

Figure 5. (Left) $\mathrm{T}_{1}-\mathrm{T}_{2}$ spectrum of $8 \%$ BSA gel in $\mathrm{D}_{2} \mathrm{O}$ and (Right) $1 \%$ pectin in $\mathrm{D}_{2} \mathrm{O}$.

One obvious way of suppressing the water signal in the model BSA-pectin system is to simply replace the water, $\mathrm{H}_{2} \mathrm{O}$, with $\mathrm{D}_{2} \mathrm{O}$ and figure 5 shows the resulting spectra of the separate component biopolymers in $\mathrm{D}_{2} \mathrm{O}$. This indeed succeeds in amplifying the biopolymer peaks though there is still a water peak (peak 1) arising from residual unexchanged protons mainly in the form of HOD. Unfortunately, however, the $\mathrm{T}_{2}$ of the main biopolymer $\mathrm{CH}$ peak (figure 5, left spectrum, peak 3) is so short (about $400 \mu \mathrm{s}$ ) that it cannot be completely characterised with the $T_{1}-T_{2}$ pulse sequence because the shortest echo time is also about $400 \mu \mathrm{s}$. The same is true of the pectin $\mathrm{CH}$ peaks which are sub-millisecond. While it may be possible to characterise the short $\mathrm{T}_{2}$ components with the $\mathrm{T}_{1}-\mathrm{T}_{2} *$ pulse sequence (where the CPMG sequence in the second dimension is replaced with an FID) this requires a well-shimmed high resolution magnet otherwise the measured $\mathrm{T}_{2}{ }^{*}$ merely reflects the magnet inhomogeneity rather than anything intrinsic to the sample. Lacking such an instrument we have not yet been able to investigate whether phase separation significantly alters the biopolymer $T_{1}-T_{2}$ peaks. Of course, if the intrinsic $T_{2}$ 's are shorter than the $T_{2} *$ of the magnet field inhomogeneity then the the $T_{1}-T_{2} *$ approach yields reliable spectra. This will usually be the case with lower water-content samples. Figure 6 shows the $\mathrm{T}_{1}-\mathrm{T}_{2}$ and $\mathrm{T}_{1}-\mathrm{T}_{2} *$ spectra of a $40 \% \mathrm{w} / \mathrm{w}$ BSA gel and it can be seen that the $T_{1}-T_{2}$ spectra characterises the long $T_{2}$ peaks; while the $T_{1}-T_{2} *$ spectra the short $\mathrm{T}_{2}$ peaks.

Another draw-back of multidimensional $T_{1}-T_{2}$ spectroscopy is the lengthy acquisition time of 2-4 hours which limits its use to stable food materials. This is a pity because it means that real-time monitoring of food processing responses, phase changes and rapid storage changes cannot be done with the existing slow form of the technique. Clearly an ultrafast $\mathrm{T}_{1}-\mathrm{T}_{2}$ pulse sequence would be highly desirable and the recent development of ultrafast multidimensional high resolution $\mathrm{NMR}^{8}$ based on slice-selection with soft RF pulses suggests that a similar approach might, in principle, achieve this. Figure 8 illustrates the idea. A conventional 1-dimensional $\mathrm{T}_{1}$ (or 2-dimensional $T_{1}-T_{2}$ ) measurement is very slow because of the need to repeatedly step out the inversion recovery time, $\mathrm{t}_{1}$, which, to avoid saturation, necessitates a delay of $5 \mathrm{~T}_{1}$ between each repetition. However, by using slice-selective RF $180^{\circ}$ 
pulses in a fixed gradient, different inversion recovery times can be associated with different slices in the sample and so

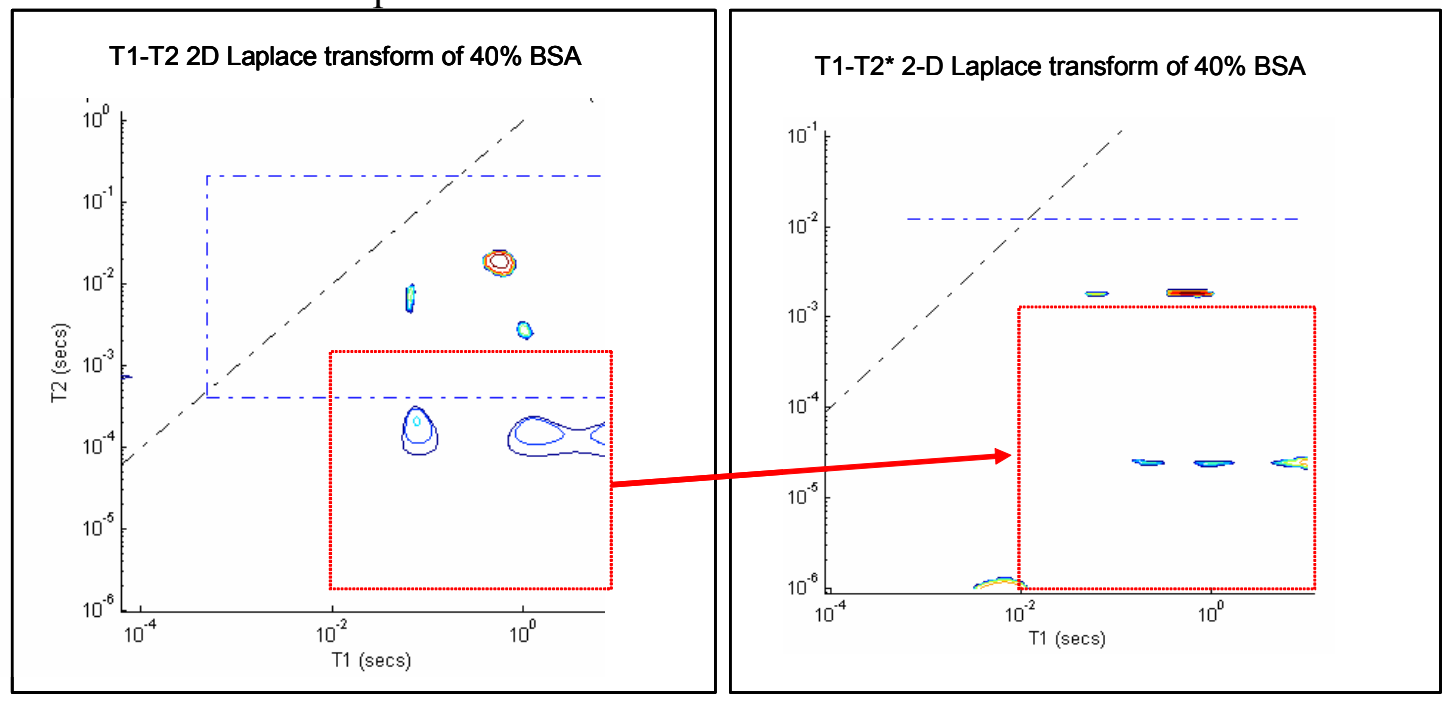

Figure 6. (Left) $T_{1}-T_{2}$ spectrum of a $40 \%$ native $B S A$ solution. (Right) $T_{1}-T_{2} *$ spectrum of the same sample.

can be acquired in a single-shot. Each slice has a different $t_{1}$ because the slice selection pulse is progressively delayed. The signal in each slice can be separately resolved by acquiring an FID (or Hahn echo with short echo time) in a constant $\mathrm{G}_{z}$ gradient. The points in the one-dimensional profile obtained by Fourier transformation are then associated with different slices and therefore different inversion recovery times, allowing $T_{1}$ to be extracted directly from the profile. If instead of a simple FID or Hahn echo, a complete CPMG echo train is acquired in the read-out gradient, then this provides a second " $t_{2}$ " dimension associated with $\mathrm{T}_{2}$ relaxation and the sequence allows the acquisition of a single-shot, ultrafast 2dimensional $\mathrm{T}_{1}-\mathrm{T}_{2}$ spectrum.

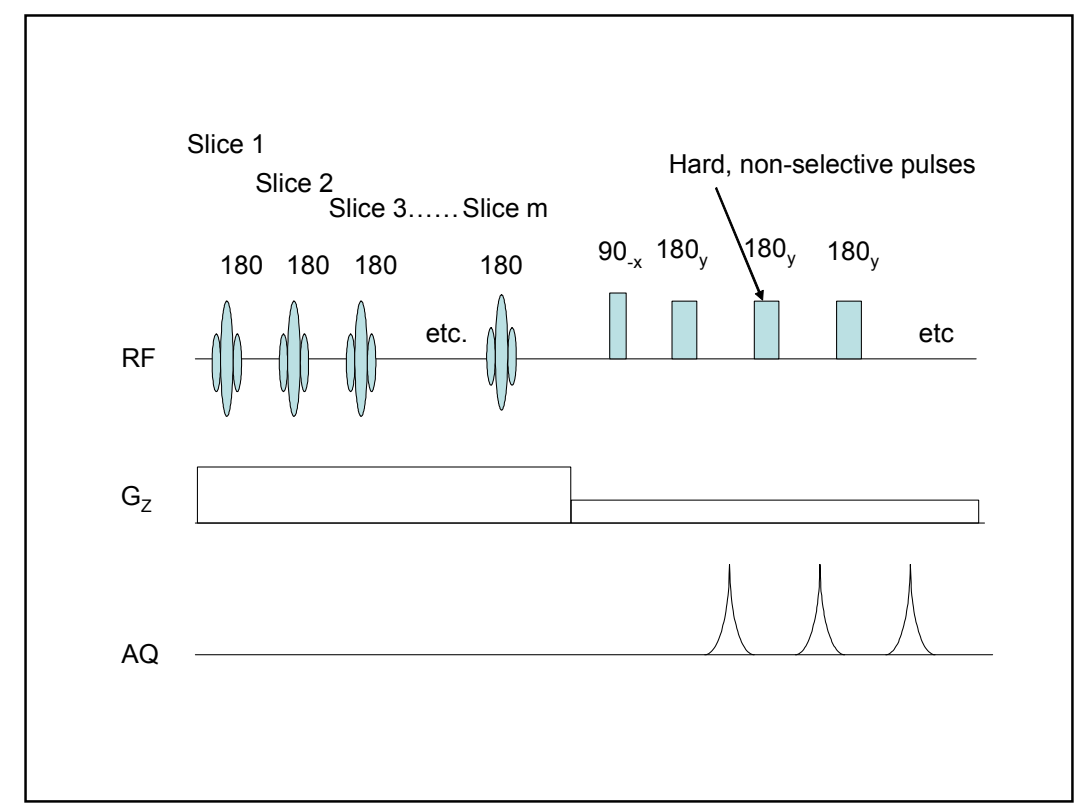

Figure 7. A possible ultrafast $T_{1}-T_{2}$ pulse sequence. 
Most real multiphase food materials cannot be made up in $\mathrm{D}_{2} \mathrm{O}$ so a more general strategy for water suppression in $\mathrm{T}_{1}-\mathrm{T}_{2}$ spectra is required. A possible ultra-fast watersuppression protocol which exploits the high diffusivity of water molecules relative to dissolved solutes, is illustrated in figure 8. It comprises three parts, namely an initial water-suppression PGSE sequence, followed by a modified pulsed gradient stimulated echo sequence and finally a CPMG sequence. The gradients in the initial PGSE part of the sequence are first adjusted to suppress the signal from the more mobile water. The stimulated echo part of the sequence in figure 8 not only provides the variable $T_{1}$ dimension but also ensures that any fresh water magnetisation created by longitudinal relaxation during the $t_{1}$ delay is spoiled by the second gradient pulse of the stimulated echo sub-sequence. Whether this succeeds in producing quantitative water-suppressed spectra remains to be investigated.

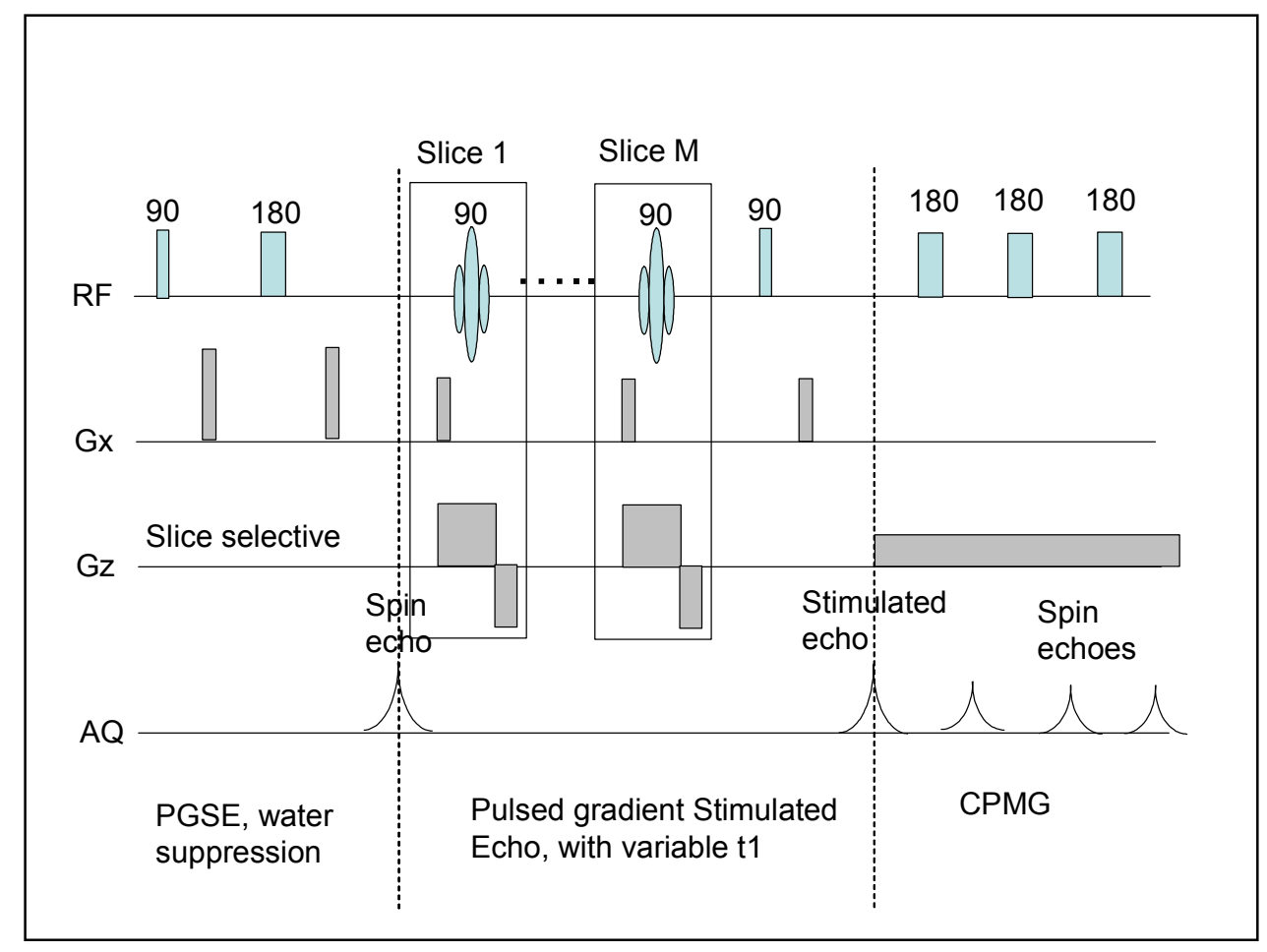

Figure 8. A possible ultra-fast, water-suppressed $T_{1}-T_{2}$ pulse sequence.

Further extensions of the multidimensional approach arise if the frequencydependence of both $T_{1}$ and $T_{2}$ are exploited. In many foods $T_{1}$ decreases with decreasing magnetic field strength, $\mathrm{B}_{0}$, (or equivalently, with decreasing resonance frequency, $\omega_{0}$, since $\omega_{0}=\gamma \mathrm{B}_{0}$ ) and this dispersive frequency behaviour is related to the spectral density of molecular motions. Likewise, in most biopolymer and food systems the $T_{2}$ of the water proton peak depends on frequency $\left(\omega_{0}\right)$ through processes such as proton exchange with exchangeable biopolymer protons. Each peak in the $T_{1^{-}}$ $\mathrm{T}_{2}$ spectrum is therefore expected to have a characteristic frequency dependence which can, in principle, be measured in fast field cycling relaxometers. In this way 2dimensional $T_{1}-T_{2}$ spectra could, perhaps, be extended into the third, frequency $\left(\omega_{0}\right)$ dimension and this would not only provide additional dynamic information but might also be useful in separating broad overlapping peaks in the 2-dimensional $T_{1}-T_{2}$ 
spectrum. Such measurements have yet to be undertaken because they require a new generation of field cycling relaxometers with high field stability. A suitable prototype relaxometer has recently been developed and should soon be available commercially.
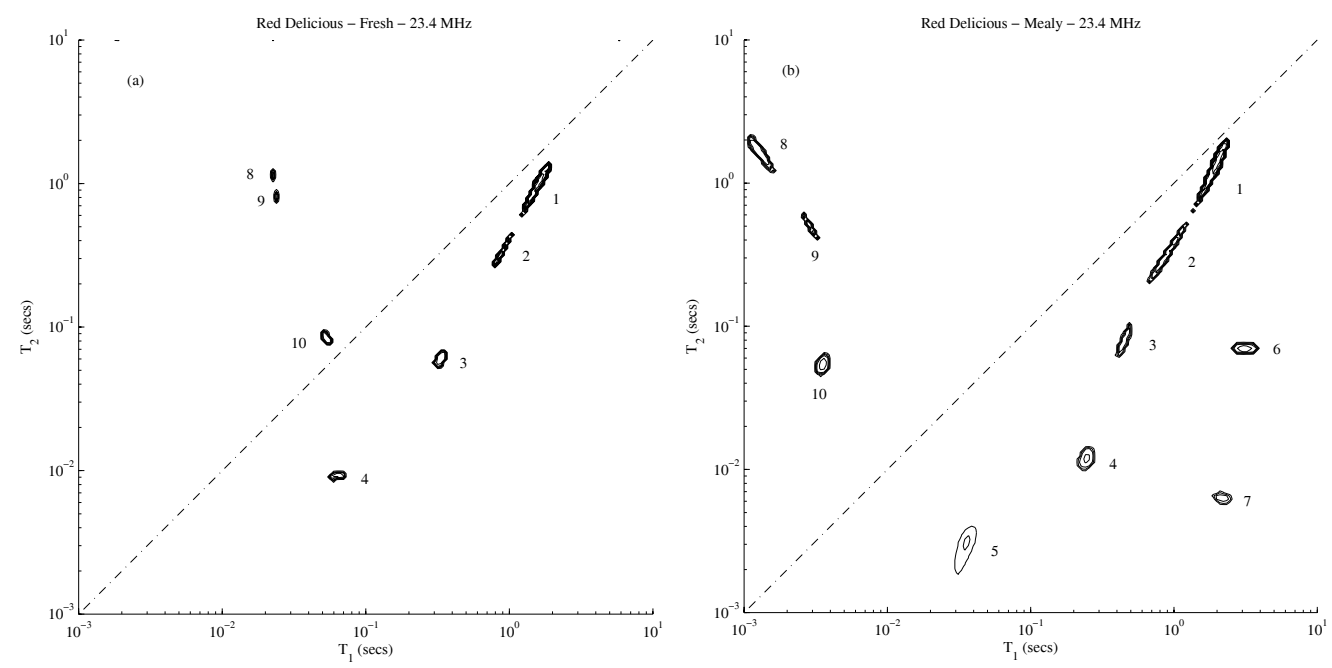

Figure 9. $T_{1}-T_{2}$ correlation spectra at $23.4 \mathrm{MHz}$ of fresh (left) and mealy (Right) apple parenchyma tissue. Provisional peak assignments: $1=$ vacuolar water; $2=$ cytoplasm; $3=$ water in starch granules; $4=$ water in cell wall region; $6=$ protein sugar and vascular tissue; $7=$ pectin.

\section{Water compartmentation in plant tissue}

$\mathrm{T}_{1}-\mathrm{T}_{2}$ methods are ideally suited for monitoring the sub-cellular redistribution of water in cellular plant tissue because diffusion between compartments is limited both by membrane permeability barriers and by the relatively large compartment sizes, in the range ten to several hundred microns. This is particularly useful because it permits a detailed analysis of the subcellular changes induced by physiological changes. Some of these, such as mealiness in apples, cause unacceptable quality loss and therefore have considerable commercial significance. To illustrate the power of multidimensional relaxometry we therefore examine is greater detail the water redistribution induced by mealiness in apples.

Mealiness is internal quality defect characterised by a lack of juiciness, soft and sandy texture when chewing and affects fruits such as apples, peaches and nectarines. Being a sensory attribute, it is difficult to be detected in the selection chain, so mealy fruit reaches the market even though its quality is very low. Mealiness is induced by inappropriate storage and is associated with a disintegration of the middle lamella to the point where cells separate instead of rupturing when a force is applied as during chewing. Figure 9 shows examples of $\mathrm{T}_{1}-\mathrm{T}_{2}$ correlation spectra for fresh and mealy apple together with peak assignments. These spectra were acquired at 23.4 MHz with a CPMG echo spacing of $400 \mu \mathrm{s}$. Based on the observation of 6 fresh apples and 7 mealy apples it was found that mealiness is associated with a transfer of water out of the vacuole into the cytoplasm and extra-cellular spaces resulting in a reduction in the vacuolar peak area from an average of $84 \%$ in the fresh to $77 \%$ in the mealy. The average $T_{1}$ and $T_{2}$ for the peak 2 , assigned to water in the cytoplasm and extra-cellular compartment, also increases with increasing mealiness $\left(T_{2}\right.$ is $267 \mathrm{~ms}$ for the fresh compared to $348 \mathrm{~ms}$ for the mealy; $\mathrm{T}_{1}$ is $579 \mathrm{~ms}$ for the fresh and $917 \mathrm{~ms}$ for the mealy). The average area of this peak also increases as mealiness develops, 
agreeing with earlier observation that water is transferred out of the vacuole into the cytoplasm and extra-cellular compartments. The longitudinal relaxation times $\left(\mathrm{T}_{1}\right)$ of peak 4 assigned to water associated with the cell wall are also consistently longer in mealy apples than those of fresh apples. This can be related to the softening of plant tissue as a result of fruit ripening and to the dissolution of cell wall polymers.
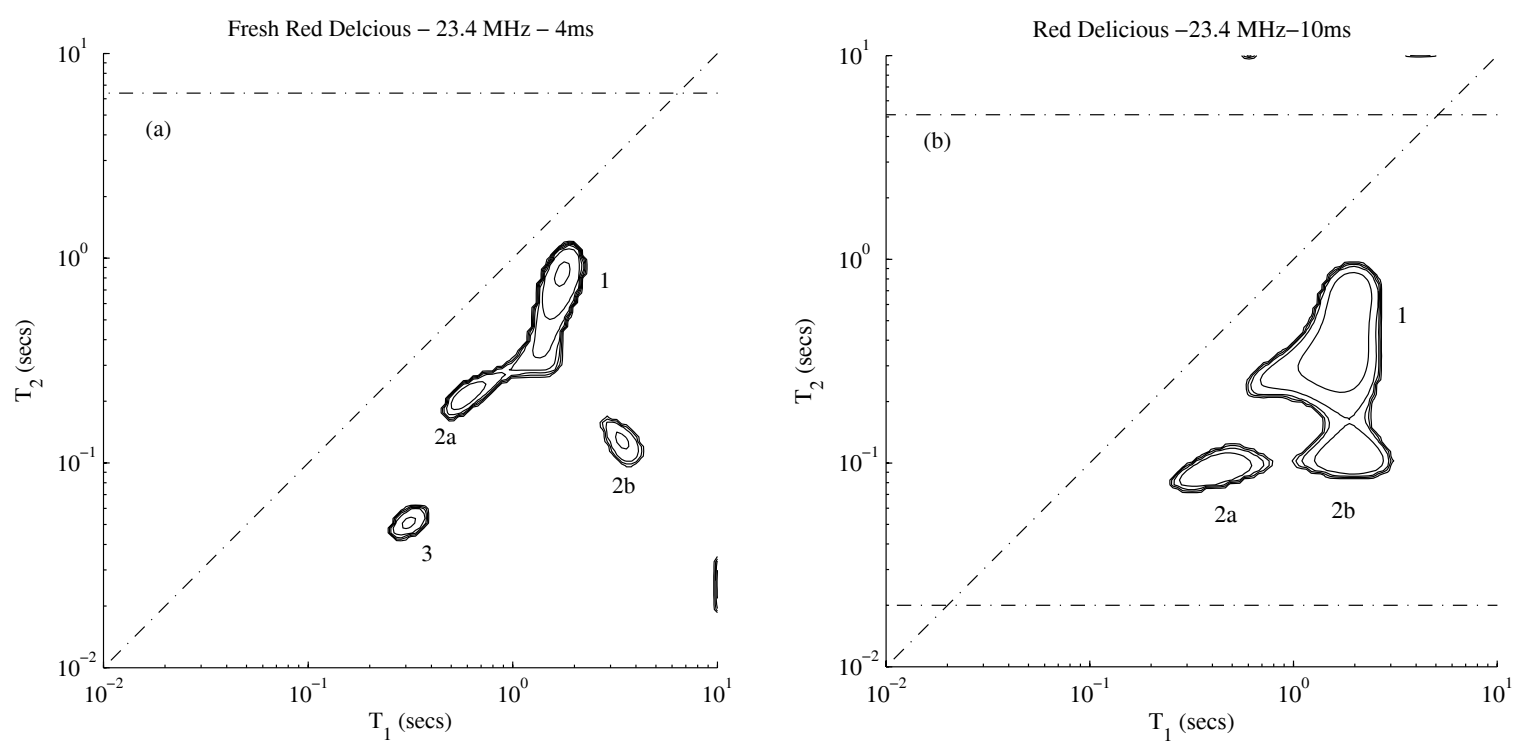

Figure 10. $T_{1}-T_{2}$ spectra for fresh apple parenchyma tissue acquired at $23.4 \mathrm{MHz}$ with CPMG pulse spacings of (left) $4 \mathrm{~ms}$ and (right) $10 \mathrm{~ms}$.

Changing the CPMG pulse spacing and the spectrometer frequency give additional information. Figure 10 shows the $\mathrm{T}_{1}-\mathrm{T}_{2}$ correlation spectra of fresh apple tissue acquired at $23.4 \mathrm{MHz}$ with two different pulse spacings. Peak 1, is assigned to the water in the vacuole has a shorter $\mathrm{T}_{2}$ at a pulse spacing of $10 \mathrm{~ms}$ and the peak is broader, spreading in the $T_{2}$ direction, and its maximum $T_{1}$ is longer than at shorter $\tau$. Peak 2, assigned to the water in the cytoplasm seems to be resolved in the $T_{1}$ domain as the pulse spacing is increased. It has a shorter $\mathrm{T}_{1}$ component (peak 2a) and another component with a longer $T_{1}$. The water signal amplitude decreases with increasing $\tau$
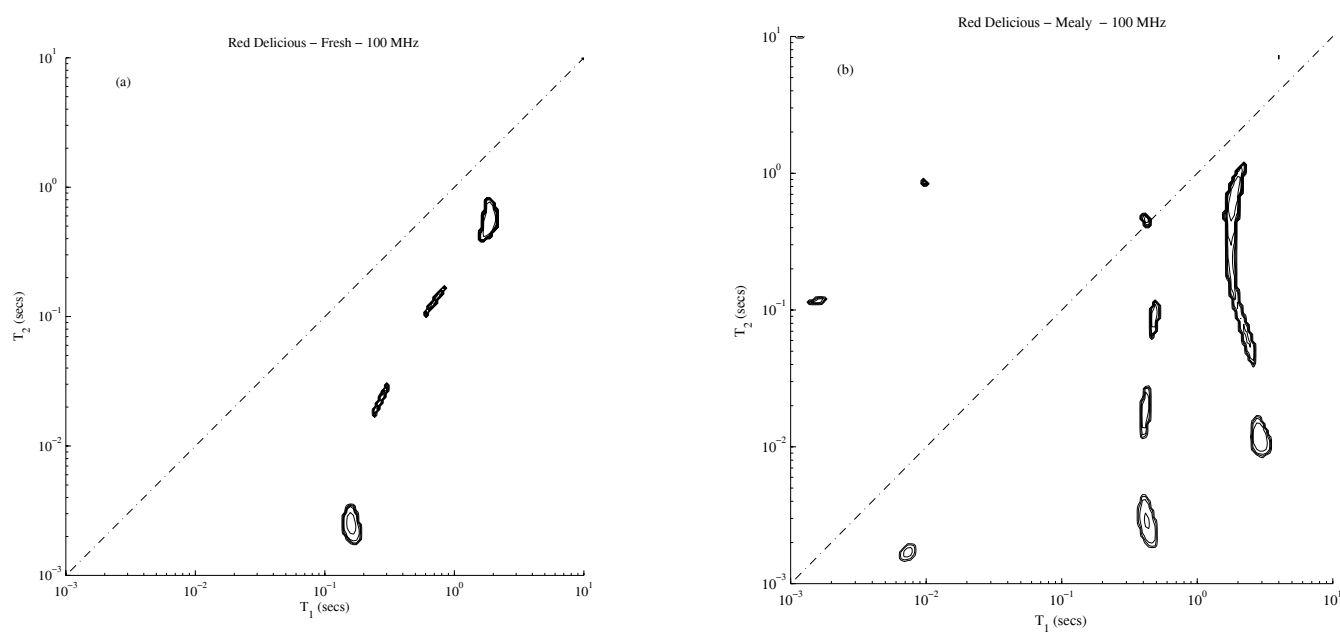

Figure 11. $T_{1}-T_{2}$ spectra of (left) fresh and (right) mealy parenchyma apple tissue acquired at a higher frequency of $100 \mathrm{MHz}$. 
so that the non-exchanging proton signal (peak 6 for example) assigned protein and sugar $\mathrm{CH}$, increases. All peaks with $\mathrm{T}_{2} \leq 4 \tau$, are unobservable so there are no peaks associated with the cell wall water at 4 and $10 \mathrm{~ms}$.

Figure 11 shows the effect of increasing the spectrometer frequency to $100 \mathrm{MHz}$ on the $T_{1}-T_{2}$ spectra of both fresh and mealy apples. The $T_{1}-T_{2}$ correlation spectra of fresh apple tissue show similar features to those acquired at $23.4 \mathrm{MHz}$ in figure $9 \mathrm{a}$. The peak with the longest relaxation times and largest area is associated with water in the vacuole. The second largest peak can be assigned to water associated with the cytoplasm and extra-cellular compartments. The spectrum of mealy apple tissue at $100 \mathrm{MHz}$ (figure 11, right) shows how the peaks associated with the vacuole and cytoplasm are stretched in the $\mathrm{T}_{2}$ dimension probably as a result of diffusion through strong internal magnetic fields created by the magnetic susceptibility difference across the air-water interface across the air spaces in the mealy tissue. This agrees with the skew histograms of mealy apples observed by Barreiro et $a l^{9}$.

Multidimensional relaxometry can also be combined with NMR pulse sequences which probe water diffusion. For example the Pulsed Gradient Spin Echo (PGSE) sequence can be combined with the CPMG sequence to give 2-dimensional $\mathrm{T}_{2}$-D cross-correlation spectroscopy. Figure 12a shows how this of this type of experiment on apple resolves the slower diffusing sucrose (peak 3). Figure 12 (right) shows the $\mathrm{T}_{2}$-D spectrum for avocado parenchyma tissue and succeeds in nicely separating the two oil peaks from the water peaks. This is especially useful because the oil and water peaks overlap in a 1-dimensional CPMG spectrum and greatly confuse the interpretation. The same $T_{2}-\mathrm{D}$ methodology could also be used for separating microscopic water populations with different degrees of restricted diffusion induced by the microstructure, but in many cases the microstructure can be determined by direct measurements of the water self-diffusion propagator, to which we now turn.
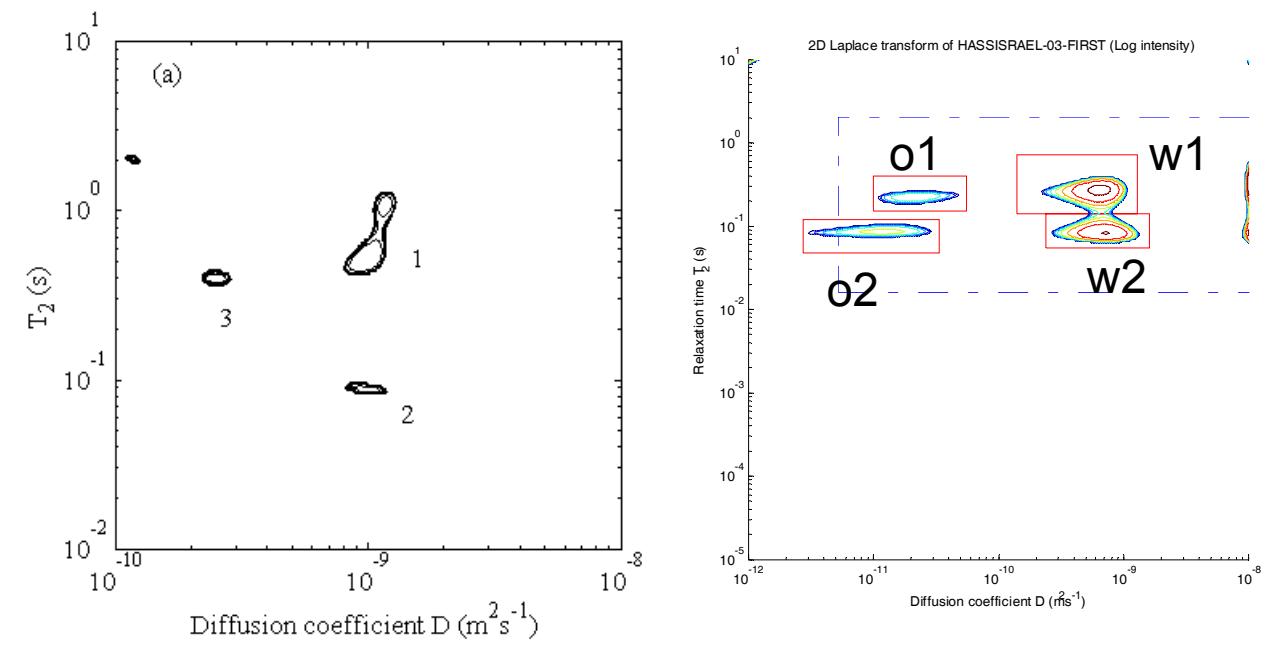

Figure 12. $T_{2}-D$ spectra of (left) parenchyma apple tissue. Peak 3 is assigned to sucrose, 1 and 2 to water. (right) Avocado tissue. o1 and o2 are oil peaks, w1 and w2 water peaks.

\section{The water self-diffusion propagator}


Although in NMR relaxometery the high mobility of water molecules leads to averaging of the signal between microscopic domains and therefore loss of morphological information, the high diffusivity can also be used as a probe of the local microstructure. The water self-diffusion propagator, or more precisely, the conditional displacement probability, $\mathrm{P}_{\mathrm{s}}\left(\mathbf{r} / \mathbf{r}^{\prime}, \mathrm{t}\right)$, is the conditional probability for finding a water molecule at location $\mathbf{r}^{\prime}$ at a time $t$ when it was originally at $\mathbf{r}$ at time zero. The conditional probability flux, $\mathrm{J}$, is obtained from the propagator as,

$\mathbf{J}=-\mathrm{D} \nabla \mathrm{P}_{\mathrm{s}}$

where D is the self-diffusion coefficient. Because the total conditional probability is conserved, the continuity equation applies,

$\nabla . \mathbf{J}=-\partial \mathrm{P}_{\mathrm{s}} / \partial \mathrm{t}$

so that, combining [17] and [18] gives

$\partial \mathrm{P}_{\mathrm{s}} / \partial \mathrm{t}=\mathrm{D} \nabla^{2} \mathrm{P}_{\mathrm{s}}$

which shows the relationship between the diffusion propagator and the self diffusion coefficient. There are many advantages to working with the self-diffusion propagator, $\mathrm{P}_{\mathrm{s}}$, rather than with the self-diffusion coefficient, D. In complex spatially microheterogeneous systems there are no analytic solutions to the diffusion equation so it is far easier to use numerical simulations based, for example, on the Monte Carlo or cellular automata methods to directly calculate $\mathrm{P}_{\mathrm{s}}$ for ensembles of water molecules randomly located in the matrix than it is to try to solve diffusion equations.

Analytic expressions for the average propagator have been derived for water diffusion in a variety of simple morphologies appropriate to microstructured foods. These include matrices of connected pores ${ }^{10}$, fractal geometries ${ }^{10}$ and multicompartment systems ${ }^{11}$. In this context the example of starch granules in a protein matrix can be modelled as a 2-compartment system where the water transport is related to the intrinsic water self diffusion coefficients in the separate starch and albumin microphases. However, in most cases, such analytical models are only crude representations of the actual sample microheterogeneity. Current research is therefore aimed at calculating the propagator numerically for realistic morphologies. A particularly promising area of current research involves numerically reconstructing the morphology of microheterogeneous matrices ${ }^{12}$. The reconstruction process involves numerically generating random matrices having the same morphological features, such as porosity and spatial correlation functions as the real system These reconstructed morphologies can then be used as templates for numerical calculation of the diffusion propagator using, for example, the finite element methods of FEMLAB ${ }^{13}$ or some other numerical approach such as the Monte Carlo or cellular automaton methods. Alternatively the actual digitised confocal images and cross-sections could be used directly in the propagator simulations.

Another motive for preferentially calculating the self-diffusion propagator rather than the diffusion coefficient, is that the self-diffusion propagator can be measured directly with NMR pulsed field gradient methods even in complex heterogeneous systems. To this we now turn.

\section{Experimental probes of the water self-diffusion propagator}


As is well known, the echo attenuation caused by water diffusion throughout the whole sample in a PGSE experiment can be written

$\mathrm{S}(\mathrm{q}, \Delta)=\iint \operatorname{drdr}^{\prime} \rho(\mathrm{r}) \mathrm{P}_{\mathrm{s}}\left(\mathbf{r} \mid \mathbf{r}^{\prime}, \Delta\right) \exp \left[\mathrm{i} 2 \pi \mathbf{q} \cdot\left(\mathbf{r}^{\prime}-\mathbf{r}\right)\right]$

where $\mathbf{q}$ is the pulsed gradient area, $\gamma \mathbf{G} \delta$ and $\gamma$ is the proton gyromagnetic ratio and $\Delta$ is a variable diffusion time. Here $\rho(r)$ is the water proton density, giving the initial distribution and the exponential factor gives the phase change, but, most significantly we see that this phase change is weighted by the self diffusion propagator, $\mathrm{P}_{\mathrm{s}}\left(\mathbf{r} \mid \mathbf{r}^{\prime}, \Delta\right)$. To take the analysis further it is usual to assume equilibrium and translational invariance (i.e. that the results are independent of the initial position of the water molecules) so that equation [4] can be transformed to the displacement variable, $\mathbf{R}=$ $\mathbf{r}^{\prime}$ - r, by introducing the average propagator,

$\mathrm{P}_{\mathrm{av}}(\mathbf{R}, \Delta)=\int \operatorname{dr} \rho(\mathrm{r}) \mathrm{P}\left(\mathbf{r} \mid \mathbf{r}^{\prime}, \Delta\right)$

With this transformation, equation [4] becomes the Fourier relationship,

$\mathrm{S}(\mathrm{q}, \Delta)=\int \mathrm{dR} \mathrm{P}_{\mathrm{av}}(\mathbf{R}, \Delta) \exp (\mathrm{iq} \cdot \mathbf{R})$

Which means that the average propagator can be obtained by inverse Fourier transformation of the NMR echo amplitude measured as a function of increasing wavevector, q:

$\mathrm{P}_{\mathrm{av}}(\mathbf{R}, \Delta)=\int \mathrm{d} \mathbf{q} \mathrm{S}(\mathbf{q}, \Delta) \exp (-\mathrm{iq} \cdot \mathbf{R})$

The resulting diffusion propagator can be compared with the theoretical calculations discussed in the previous section. Alternatively, the calculated propagator can be inserted into equation [6] and the resulting echo amplitude, $\mathrm{S}(\mathrm{q}, \Delta)$ compared with experiment. So far the discussion of the diffusion propagator has been "onedimensional" but, just like multidimensional relaxometry, much more information can be obtained by considering multidimensional diffusometry ${ }^{14}$. Indeed, 2dimensional sequences such as $\mathrm{D}_{1}-\mathrm{D}_{2}$ spectroscopy obtained by concatenating two PGSE sequences provide further insight into the anisotropic microscopic diffusion of water in macroscopically isotropic media.

\section{Conclusion}

Multidimensional relaxometry and diffusometry are powerful new tools to the NMR armoury for probing the microscopic distribution of water in complex heterogeneous food matrices. In cellular plant tissue these new tools succeed in resolving the subcellular compartmentation of water and can be used to examine the effects of processing, storage and physiological changes on the water distribution of the tissue. The microphases of mixed biopolymer systems can also be resolved provided there is sufficient difference in relaxation times and the diffusion length is sufficient to prevent diffusive averaging of the signal. This in practice limits the spatial resolution in high water content systems to 100 microns or more. Nevertheless there is clearly a great deal more development work required before the power of 
multidimensional NMR relaxometry and diffusometry is fully realised as a probe of microscopic water distribution in complex food systems.

Although the discussion has focused on water in food, many of the same issues arise in non-food materials. A detailed knowledge of the factors controlling water transport in synthetic hydrogels is essential for the rational development of new contact lens materials and medical implants, where desired mechanical properties have to be combined with specified moisture, gas and salt permeability. At present the research is an empirical trial-and-error exercise involving the labour-intensive synthesis of numerous hydrogels of differing molecular composition and nanopore structure many of which fail the performance criteria. In like manner, water transport through porous rock plays a vital role in the oil industry where brine is used to extract oil from underground reservoirs. The relationship between water transport and the pore structure and connectivity in rock has therefore been an active research topic for many years. It is a daunting thought that water transport in food is even more complex than in porous rock because, unlike rock, the food biopolymer matrix is not rigid but has a structure and dynamic state that depends on temperature, composition, water content and processing history.

\section{References}

1. Hills, B.P., 'Magnetic Resonance Imaging in Food Science', John Wiley and Sons, New York, 1998.

2. For example, Coulson, J.M., and Richardson, J.F., Chemical Engineering, volume 1, Fluid flow, Heat transfer and Mass Transfer, Pergamon Press, Oxford, 1993.

3. Crank, J., The mathematics of diffusion, Oxford Science Publications, Clarendon Press, Oxford, 1993.

4. Tang, H, and Hills, B P, The distribution of water in native starch granules- A multinuclear approach, Carbohydrate polymers, 2000, 43, 375-387.

5. Tang, H, and Hills, B P, A multinuclear NMR study of the gelatinization and acid hydrolysis of native potato starch, Carbohydrate Polymers, 2001, 46, 7-18

6. Donato, L., Garnier, C., Novales, B., Durand, S., and Doublier, J.-L., Heatinduced gelation of BSA/Low-methoxy Pectin systems and the effect of calcium ions. Biomacromolecules, 2005, 6, 374-385.

7. Song, Y.-Q, Venkataramanan, L, Hurlimann, M D., Flaum, M, Frulla, P, and Straley, $\mathrm{C}, \mathrm{T}_{1}-\mathrm{T}_{2}$ correlation spectra obtained using a fast two-dimensional Laplace Inversion, Journal of Magnetic Resonance 2002, 154, 261-268,.

8. Tal,A., Shapira, B. and Frydman,L, The acquisition of multidimensional NMR spectra within a single scan, J.Magn. Reson., 2005, 176, 107

9. Barreiro P.; Moya A.; Correa E.; Ruiz-Altisent M.; Fernández-Valle M., Peirs A.; Wright K.M. and Hills B.P. Prospects for the rapid detection of mealiness in Apples by non-destructive NMR Relaxometry. Applied Magnetic Resonance (2002) 22, 387-400.

10. Callaghan, P T, Principles of NMR microscopy, Clarendon Press, Oxford, 1991

11. Karger, J, Pfeifer, H, and Heink, W, Principles and application of self-diffusion measurements by NMR, Advances in Magnetic Resonance, 1988, 12, 1-30.

12. Adler, P M, Porous Media, Butterworth-Heinemann, Boston, 1992.

13. FEMLAB software for finite element modelling can be found at www.comsol.com 
14. Callaghan, P T, Godefroy, S, and Ryland, B N, Use of the second dimension in PGSE NMR studies of porous media, Magnetic Resonance Imaging, 2003, 21, 243-248. 